\title{
A CONSTRUCTIVE METHOD TO GET RIGHT-LEFT EQUIVALENCE FOR SMOOTH MAP GERMS AND ITS APPLICATION TO DIVERGENT DIAGRAMS
}

\section{Takashi Nishimura}

Let $f, g:\left(\mathbb{R}^{n}, 0\right) \rightarrow\left(\mathbb{R}^{p}, 0\right)$ be two $C^{\infty}$ map germs. We say that $f$ and $g$ are right-left equivalent if there exist $C^{\infty}$ diffeomorphic map germs $h:\left(\mathbb{R}^{n}, 0\right) \rightarrow$ $\left(\mathbb{R}^{n}, 0\right)$ and $H:\left(\mathbb{R}^{p}, 0\right) \rightarrow\left(\mathbb{R}^{p}, 0\right)$ such that the following diagram commutes:

$$
\begin{array}{ccc}
\left(\mathbb{R}^{n}, 0\right) & \stackrel{f}{\longrightarrow} & \left(\mathbb{R}^{p}, 0\right) \\
h \downarrow & & H \downarrow \\
\left(\mathbb{R}^{n}, 0\right) & \stackrel{g}{\longrightarrow} & \left(\mathbb{R}^{p}, 0\right) .
\end{array}
$$

This equivalence relation seems to be the most natural equivalence relation because this says that $f$ coincides with $g$ under suitable coordinate transformations of the source and the target spaces.

In $[\mathrm{N}]$, the author proposed a method to construct $C^{\infty}$ diffeomorphic map germs which give right-left equivalence for the given two $C^{\infty}$ map germs and got several extensions of the known results concerning right-left equivalence.

A $C^{\infty}$ divergent diagram $\left(f_{1}, f_{2}\right):\left(\mathbb{R}^{p}, 0\right) \leftarrow\left(\mathbb{R}^{n}, 0\right) \rightarrow\left(\mathbb{R}^{q}, 0\right)$ is a pair of $C^{\infty}$ map germs $f_{1}:\left(\mathbb{R}^{n}, 0\right) \rightarrow\left(\mathbb{R}^{p}, 0\right)$ and $f_{2}:\left(\mathbb{R}^{n}, 0\right) \rightarrow\left(\mathbb{R}^{q}, 0\right)$. We often identify a $C^{\infty}$ divergent diagram germ $\left(f_{1}, f_{2}\right):\left(\mathbb{R}^{p}, 0\right) \leftarrow\left(\mathbb{R}^{n}, 0\right) \rightarrow\left(\mathbb{R}^{q}, 0\right)$ with the $C^{\infty}$ map germ $f:\left(\mathbb{R}^{n}, 0\right) \rightarrow\left(\mathbb{R}^{p} \times \mathbb{R}^{q},(0,0)\right)$ such that $f(x)=$ $\left(f_{1}(x), f_{2}(x)\right)$ and we write as $f=\left(f_{1}, f_{2}\right)$. Let $f=\left(f_{1}, f_{2}\right), g=\left(g_{1}, g_{2}\right)$ : $\left(\mathbb{R}^{p}, 0\right) \leftarrow\left(\mathbb{R}^{n}, 0\right) \rightarrow\left(\mathbb{R}^{q}, 0\right)$ be two $C^{\infty}$ divergent diagrams. We say $f$ and $g$ are equivalent as divergent diagrams if there exist $C^{\infty}$ diffeomorphic map germs $h:\left(\mathbb{R}^{n}, 0\right) \rightarrow\left(\mathbb{R}^{n}, 0\right), H_{1}:\left(\mathbb{R}^{p}, 0\right) \rightarrow\left(\mathbb{R}^{p}, 0\right)$ and $H_{2}:\left(\mathbb{R}^{q}, 0\right) \rightarrow\left(\mathbb{R}^{q}, 0\right)$ such 
that the following diagram commutes:

$$
\begin{array}{ccccc}
\left(\mathbb{R}^{p}, 0\right) & \stackrel{f_{1}}{\longleftarrow} & \left(\mathbb{R}^{n}, 0\right) & \stackrel{f_{2}}{\longrightarrow} & \left(\mathbb{R}^{q}, 0\right) \\
H_{1} \downarrow & & h \downarrow & & H_{2} \downarrow \\
\left(\mathbb{R}^{p}, 0\right) & \stackrel{g_{1}}{\longleftarrow} & \left(\mathbb{R}^{n}, 0\right) & \stackrel{g_{2}}{\longrightarrow} & \left(\mathbb{R}^{q}, 0\right) .
\end{array}
$$

This equivalence relation also seems to be the most natural equivalence relation for smooth divergent diagrams. However, this equivalence relation is known to be too tight to expect theorems reflecting the principle "an infinitesimal relation corresponding to this equivalence relation implies a geometric notion corresponding to this equivalence relation" for general $C^{\infty}$ divergent diagrams (for instance, see [D1, D2, Na1, Na2]). Thus, we introduce the following two weaker equivalence relations.

Definition 0.1: Let $f=\left(f_{1}, f_{2}\right), g=\left(g_{1}, g_{2}\right):\left(\mathbb{R}^{n}, 0\right) \rightarrow\left(\mathbb{R}^{p} \times \mathbb{R}^{q}, 0\right)$ be two $C^{\infty}$ divergent diagrams. We say $f$ and $g$ are equivalent over varieties if there exist $C^{\infty}$ diffeomorphic map germs $h:\left(\mathbb{R}^{n}, 0\right) \rightarrow\left(\mathbb{R}^{n}, 0\right), H_{1}:\left(\mathbb{R}^{p}, 0\right) \rightarrow$ $\left(\mathbb{R}^{p}, 0\right)$ and $H_{2}:\left(\mathbb{R}^{q}, 0\right) \rightarrow\left(\mathbb{R}^{q}, 0\right)$ having the following two properties:

(0.1.1) the following diagram commutes:

$$
\begin{array}{ccc}
\left(f_{2}^{-1}(0), 0\right) & \stackrel{f_{1}}{\longrightarrow} & \left(\mathbb{R}^{p},(0,0)\right) \\
h \downarrow & & H_{1} \downarrow \\
\left(g_{2}^{-1}(0), 0\right) & \stackrel{g_{1}}{\longrightarrow} & \left(\mathbb{R}^{p},(0,0)\right) .
\end{array}
$$

(0.1.2) the following diagram also commutes:

$$
\begin{array}{ccc}
\left(f_{1}^{-1}(0), 0\right) & \stackrel{f_{2}}{\longrightarrow} & \left(\mathbb{R}^{q},(0,0)\right) \\
h \downarrow & & H_{2} \downarrow \\
\left(g_{1}^{-1}(0), 0\right) & \stackrel{g_{2}}{\longrightarrow} & \left(\mathbb{R}^{q},(0,0)\right) .
\end{array}
$$

Definition 0.2: $\left[\right.$ AGV] Let $f=\left(f_{1}, f_{2}\right), g=\left(g_{1}, g_{2}\right):\left(\mathbb{R}^{n}, 0\right) \rightarrow\left(\mathbb{R}^{p} \times \mathbb{R}^{q}, 0\right)$ be two $C^{\infty}$ divergent diagrams. We say $f$ and $g$ are $\mathcal{R}^{+}$-equivalent if there exist $C^{\infty}$ diffeomorphic map germs $h:\left(\mathbb{R}^{n}, 0\right) \rightarrow\left(\mathbb{R}^{n}, 0\right)$ and $H:\left(\mathbb{R}^{p} \times \mathbb{R}^{q},(0,0)\right) \rightarrow$ $\left(\mathbb{R}^{p} \times \mathbb{R}^{q},(0,0)\right)$ having the following two properties:
(0.2.1) the following diagram commutes:

$$
\begin{array}{ccc}
\left(\mathbb{R}^{n}, 0\right) & \stackrel{f}{\longrightarrow} & \left(\mathbb{R}^{p} \times \mathbb{R}^{q},(0,0)\right) \\
h \downarrow & & H \downarrow \\
\left(\mathbb{R}^{n}, 0\right) & \stackrel{g}{\longrightarrow} & \left(\mathbb{R}^{p} \times \mathbb{R}^{q},(0,0)\right),
\end{array}
$$

(0.2.2) $\quad H$ has the following form

$$
H\left(y, y^{\prime}\right)=\left(y+H_{1}\left(y^{\prime}\right), H_{2}\left(y^{\prime}\right)\right)
$$

where $y \in \mathbb{R}^{p}$ and $y^{\prime} \in \mathbb{R}^{q}$.

In this paper, first we will review our method of [N] (in §1), and then we will apply this method to $C^{\infty}$ divergent diagrams.

Main results in this paper are estimates of the order of determinacy of the given $C^{\infty}$ divergent diagrams with respect to equivalence over varieties and $\mathcal{R}^{+}$. equivalence (theorems (2.1) and (2.2) in $\S 2$ ). $\S \S 3-6$ will be devoted to the proof of theorems (2.1) and (2.2).

\section{Review of Our Method}

We review how to get $C^{\infty}$ diffeomorphic map germs which gives the right-left equivalence for the given two $C^{\infty}$ map germs.

Let $f, g:\left(\mathbb{R}^{n}, 0\right) \rightarrow\left(\mathbb{R}^{p}, 0\right)$ be two $C^{\infty}$ map germs. We say $f$ and $g$ are $\mathcal{K}$-equivalent if there exist a $C^{\infty}$ diffeomorphic map germ $h:\left(\mathbb{R}^{n}, 0\right) \rightarrow\left(\mathbb{R}^{n}, 0\right)$ and a $C^{\infty}$ map germ $M:\left(\mathbb{R}^{n}, 0\right) \rightarrow(G L(p, \mathbb{R}), M(0))$ such that $f(x)=$ $M(x) g(h(x))$. $\mathcal{K}$-equivalence is also an equivalence relation for $C^{\infty}$ map germs, which was introduced by J.Mather([M1]). This $\mathcal{K}$-equivalence relation is relatively easier to treat than right-left equivalence. For instance, if both of $f$ and $g$ are finitely determined with respect to $\mathcal{K}$-equivalence, then $f$ and $g$ are $\mathcal{K}$-equivalent if, and only if, their local algebras $\mathcal{E}_{x} /<f>$ and $\left.\mathcal{E}_{x} /<g\right\rangle$ are isomorphic as $\mathbb{R}$-algebras; where $\mathcal{E}_{x}$ is the $\mathbb{R}$-algebra of all $C^{\infty}$ function 
germs $\left(\mathbb{R}^{n}, 0\right) \rightarrow \mathbb{R}$ and $\left\langle f>\right.$ and $\left\langle g>\right.$ are the ideals in $\mathcal{E}_{x}$ generatetd by component functions of $f$ and $g$ respectively (see [M2]).

Our method starts from judging whether or not $f$ and $g$ are $\mathcal{K}$-equivalent. Since $\mathcal{K}$-equivalence is a weaker equivalence relation than right-left equivalence, we see if $f$ and $g$ are not $\mathcal{K}$-equivalent, then $f$ and $g$ are not right-left equivalent.

From now on in this chapter, we assume that $f$ and $g$ are $\mathcal{K}$-equivalent. Namely,

Assumption 1.1: Suppose that there exist $C^{\infty}$ diffeomorphic map germ $s$ : $\left(\mathbb{R}^{n}, 0\right) \rightarrow\left(\mathbb{R}^{n}, 0\right)$ and a $C^{\infty}$ map germ $M:\left(\mathbb{R}^{n}, 0\right) \rightarrow(G L(p, \mathbb{R}), M(0))$ such that $f(x)=M(x) g(s(x))$.

Next, we consider the $C^{\infty}$ deformation $F:\left(\mathbb{R}^{n} \times \mathbb{R}^{p},(0,0)\right) \rightarrow\left(\mathbb{R}^{p}, 0\right)$ of $f$ having the following form:

$$
\begin{aligned}
F(x, \lambda) & =M(x)(g(s(x))-\lambda) \\
& =f(x)-M(x) \lambda .
\end{aligned}
$$

We concentrate on the case when $F$ has a triviality. Namely, our second assumption is the following (1.2). We treat two kinds of $p$-dimensional euclidean space $\mathbb{R}^{p}$. When we are considering $\mathbb{R}^{p}$ as the target space, we write it $\mathbb{R}_{y}^{p}$. When we are considering $\mathbb{I}^{p}$ as the parameter space, we write it $\mathbb{R}_{\lambda}^{p}$.

Assumption 1.2: Suppose that there exist $C^{\infty}$ diffeomorphic map germs $h$ : $\left(\mathbb{R}^{n} \times \mathbb{R}_{\lambda}^{p},(0,0)\right) \rightarrow\left(\mathbb{R}^{n} \times \mathbb{R}_{\lambda}^{p},(0,0)\right), H:\left(\mathbb{R}_{y}^{p} \times \mathbb{R}_{\lambda}^{p},(0,0)\right) \rightarrow\left(\mathbb{R}_{y}^{p} \times \mathbb{R}_{\lambda}^{p},(0,0)\right)$ and $\phi:\left(\mathbb{R}_{\lambda}^{p}, 0\right) \rightarrow\left(\mathbb{R}_{\lambda}^{p}, 0\right)$ such that the following diagram commutes:

$$
\begin{array}{ccccc}
\left(\mathbb{R}^{n} \times \mathbb{R}_{\lambda}^{p},(0,0)\right) & \stackrel{\left(F, \pi_{2}\right)}{\longrightarrow} & \left(\mathbb{R}_{y}^{p} \times \mathbb{R}_{\lambda}^{p},(0,0)\right) & \stackrel{\pi_{2}}{\longrightarrow} & \left(\mathbb{R}_{\lambda}^{p}, 0\right) \\
h \downarrow & & H \downarrow & \phi \downarrow \\
\left(\mathbb{R}^{n} \times \mathbb{R}_{\lambda}^{p},(0,0)\right) & \stackrel{\left(f, \pi_{2}\right)}{\longrightarrow} & \left(\mathbb{R}_{y}^{p} \times \mathbb{R}_{\lambda}^{p},(0,0)\right) & \stackrel{\pi_{2}}{\longrightarrow} & \left(\mathbb{R}_{\lambda}^{p}, 0\right) .
\end{array}
$$

Here, $\pi_{2}$ is the canonical projection to the second element. From the commutativity, we may write

$$
h(x, \lambda)=\left(h_{1}(x, \lambda), \phi(\lambda)\right) \text { and } H(y, \lambda)=\left(H_{1}(y, \lambda), \phi(\lambda)\right) .
$$

Then, we set $\phi_{H}^{\prime}:\left(\mathbb{R}_{\lambda}^{p}, 0\right) \rightarrow\left(\mathbb{R}_{y}^{p}, 0\right) \quad$ as

$$
\phi_{H}^{\prime}(\lambda)=H_{1}(0, \lambda) \text {. }
$$

The map germ $\phi_{H}^{\prime}$ is a parameter map germ from the parameter space of the deformation $F:\left(\mathbb{R}^{n} \times \mathbb{R}_{\lambda}^{p},(0,0)\right) \rightarrow\left(\mathbb{R}_{y}, 0\right)$ to the parameter space of the graph deformation of $f$. We also set $\quad \phi_{h}^{\prime}:\left(\mathbb{R}^{n}, 0\right) \rightarrow\left(\mathbb{R}^{n}, 0\right)$ as

$$
\phi_{h}^{\prime}(x)=h_{1}(x, g(s(x))) \text {. }
$$

Then we can get easily the following equality of map germs (see [N]).

$$
f\left(\phi_{h}^{\prime}(x)\right)=\phi_{H}^{\prime}(g(s(x))) \text {. }
$$

Finally, we add one more assumption.

Assumption 1.3: Suppose that the map germ $\phi_{H}^{\prime}:\left(\mathbb{R}_{\lambda}^{p}, 0\right) \rightarrow\left(\mathbb{R}_{y}^{p}, 0\right)$ is $C^{\infty}$ diffeomorphic.

Then we can prove that the map germ $\quad \phi_{h}^{\prime}:\left(\mathbb{R}^{n}, 0\right) \rightarrow\left(\mathbb{R}^{n}, 0\right) \quad$ is also $C^{\infty}$ diffeomorphic (see $[\mathrm{N}]$ ). Thus, we see

Lemma 1.4: Under the assumptions (1.1), (1,2) and (1.3), $f$ and $g$ are rightleft equivalent.

By using our method, we can prove the following Gaffney type estimate of the order of determinacy (see $[\mathrm{N}]$ ).

Theorem 1.5: Let $f:\left(\mathbb{R}^{n}, 0\right) \rightarrow\left(\mathbb{R}^{p}, 0\right)$ be a $C^{\infty}$ map germ. Suppose there exist positive integers $k$ and $\ell$ such that the following two inclusions hold:

$$
m_{x}^{k} \mathcal{E}_{x}^{p} \subset t f\left(m_{x} \mathcal{E}_{x}^{n}\right)+\omega f\left(m_{y} \mathcal{E}_{y}^{p}\right)
$$

and

$$
m_{x}^{\ell} \mathcal{E}_{x}^{p} \subset t f\left(m_{x} \mathcal{E}_{x}^{n}\right)+f^{*} m_{y} \mathcal{E}_{x}^{p}
$$

Then $f$ is $(k+\ell-1)$-determined with respect to right-left equivalence. 
Theorem (1.5) includes the following du Plessis-Wall's estimate of the order of determinacy

Corollary 1.6: $[([\mathrm{dP}, \mathbf{W}])]$ Let $f:\left(\mathbb{R}^{n}, 0\right) \rightarrow\left(\mathbb{R}^{p}, 0\right)$ be a $C^{\infty}$ map germ. Suppose there exists a positive integer $k$ such that the following inclusion holds:

$$
m_{x}^{k} \mathcal{E}_{x}^{p} \subset t f\left(m_{x} \mathcal{E}_{x}^{n}\right)+\omega f\left(m_{y} \mathcal{E}_{y}^{p}\right)
$$

Then $f$ is $(2 k-1)$-determined with respect to right-left equivalence.

\section{Estimates of the order of determinacy of a $C^{\infty}$ diver- gent diagram}

Let $f=\left(f_{1}, f_{2}\right):\left(\mathbb{R}^{n}, 0\right) \rightarrow\left(\mathbb{R}^{p} \times \mathbb{R}^{q},(0,0)\right)$ be a $C^{\infty}$ divergent diagram. For a positive integer $k$, we say $f$ is $k$-determined with respect to equivalence over varieties (resp. $\mathcal{R}^{+}$-equivalence) if for any $C^{\infty}$ divergent diagram $g=\left(g_{1}, g_{2}\right)$ : $\left(\mathbb{R}^{n}, 0\right) \rightarrow\left(\mathbb{R}^{p} \times \mathbb{R}^{q},(0,0)\right)$ such that $j^{k} g(0)=j^{k} f(0)\left(j^{k} f(0)\right.$ means the $k$-jet of $f$ at the origin), $g$ is equivalent over varieties (resp. $\mathcal{R}^{+}$-equivalent) to $f$. The letter $y$ (resp. $y^{\prime}$ ) means a point of $\mathbb{R}^{p}$ (resp. $\mathbb{R}^{q}$ ). For instance, $\mathcal{E}_{y, y^{\prime}}$ (resp. $\left.\mathcal{E}_{y^{\prime}}\right)$ is the set of all $C^{\infty}$ function germs $\left(\mathbb{R}_{y}^{p} \times \mathbb{R}_{y^{\prime}}^{q},(0,0)\right) \rightarrow \mathbb{R}$ (resp. $\left.\left(\mathbb{R}_{y^{\prime}}^{q}, 0\right) \rightarrow \mathbb{R}\right)$.

Theorem 2.1: Let $f=\left(f_{1}, f_{2}\right):\left(\mathbb{R}^{n}, 0\right) \rightarrow\left(\mathbb{R}^{p} \times \mathbb{R}^{q},(0,0)\right)$ be a $C^{\infty}$ divergent diagram. Suppose there exist positive integers $k$ and $\ell$ such that the following two inclusions hold:

$$
m_{x}^{k} \mathcal{E}_{x}^{p+q} \subset t f\left(m_{x} \mathcal{E}_{x}^{n}\right)+\omega\left(f_{1}, f_{2}\right)\left(m_{y} \mathcal{E}_{y, y^{\prime}}^{p} \oplus m_{y^{\prime}} \mathcal{E}_{y, y^{\prime}}^{q}\right)
$$

and

$$
m_{x}^{\ell} \mathcal{E}_{x}^{p+q} \subset t f\left(m_{x} \mathcal{E}_{x}^{n}\right)+\left(f_{1}, f_{2}\right)^{*} m_{y, y^{\prime}} \mathcal{E}_{x}^{p+q} .
$$

Then $f$ is $(k+\ell-1)$-determined with respect to equivalence over varieties.

Theorem 2.2: Let $f=\left(f_{1}, f_{2}\right):\left(\mathbb{R}^{n}, 0\right) \rightarrow\left(\mathbb{R}^{p} \times \mathbb{R}^{q},(0,0)\right)$ be a $C^{\infty}$ divergent diagram. Suppose there exist positive integers $k$ and $\ell$ such that the following two inclusions hold:

$$
m_{x}^{k} \mathcal{E}_{x}^{p+q} \subset t f\left(m_{x} \mathcal{E}_{x}^{n}\right)+\omega f_{2}\left(m_{y^{\prime}} \mathcal{E}_{y^{\prime}}^{p+q}\right)
$$

and

$$
m_{x}^{\ell} \mathcal{E}_{x}^{p+q} \subset t f\left(m_{x} \mathcal{E}_{x}^{n}\right)+f_{2}^{*} m_{y^{\prime}} \mathcal{E}_{x}^{p+q} .
$$

Then $f$ is $(k+\ell-1)$-determined with respect to $\mathcal{R}^{+}$-equivalence.

Theorem (2.1) includes the following corollary (2.3).

Corollary 2.3: Let $f=\left(f_{1}, f_{2}\right):\left(\mathbb{R}^{n}, 0\right) \rightarrow\left(\mathbb{R}^{p} \times \mathbb{R}^{q},(0,0)\right)$ be a $C^{\infty}$ divergent diagram. Suppose there exists a positive integer $k$ such that the following inclusion holds:

$$
m_{x}^{k} \mathcal{E}_{x}^{p+q} \subset t f\left(m_{x} \mathcal{E}_{x}^{n}\right)+\omega\left(f_{1}, f_{2}\right)\left(m_{y} \mathcal{E}_{y, y^{\prime}}^{p} \oplus m_{y^{\prime}} \mathcal{E}_{y, y^{\prime}}^{q}\right)
$$

Then $f$ is $(2 k-1)$-determined with respect to equivalence over varieties.

Theorem (2.2) includes the following corollary (2.4).

Corollary 2.4: Let $f=\left(f_{1}, f_{2}\right):\left(\mathbb{R}^{n}, 0\right) \rightarrow\left(\mathbb{R}^{p} \times \mathbb{R}^{q},(0,0)\right)$ be a $C^{\infty}$ divergent diagram. Suppose there exists a positive integer $k$ such that the following inclusion holds:

$$
m_{x}^{k} \mathcal{E}_{x}^{p+q} \subset t f\left(m_{x} \mathcal{E}_{x}^{n}\right)+\omega f_{2}\left(m_{y^{\prime}} \mathcal{E}_{y^{\prime}}^{p+q}\right) .
$$

Then $f$ is $(2 k-1)$-determined with respect to $\mathcal{R}^{+}$-equivalence.

Example 2.5: The following two examples are taken from [HIIY]. In both examples, we are setting $n=2, p=1$ and $q=2$.

$$
f=\left(f_{1}, f_{2}\right)=\left(x_{1}+x_{2},\left(x_{1}, x_{2}^{2}\right)\right) .
$$

Calculations show that

$$
m_{x}^{2} \mathcal{E}_{x}^{3} \subset t f\left(m_{x} \mathcal{E}_{x}^{2}\right)+\omega\left(f_{1}, f_{2}\right)\left(m_{y} \mathcal{E}_{y, y^{\prime}} \oplus m_{y^{\prime}} \mathcal{E}_{y, y^{\prime}}^{2}\right)
$$


and

$$
m_{x} \mathcal{E}_{x}^{3} \subset t f\left(m_{x} \mathcal{E}_{x}^{2}\right)+\left(f_{1}, f_{2}\right)^{*} m_{y, y^{\prime}} \mathcal{E}_{x}^{3} .
$$

Thus, by theorem (2.1), $f=\left(f_{1}, f_{2}\right)$ is 2-determined with respect to equivalence over varieties.

$$
f=\left(f_{1}, f_{2}\right)=\left(x_{2}+\phi\left(x_{1}, x_{2}^{3}+x_{1} x_{2}\right),\left(x_{1}, x_{2}^{3}+x_{1} x_{2}\right)\right)
$$

where $\phi:\left(\mathbb{R}^{2}, 0\right) \rightarrow(\mathbb{R}, 0)$ is an arbitrary $C^{\infty}$ function germ. Calculations show that

$$
m_{x}^{3} \mathcal{E}_{x}^{3} \subset t f\left(m_{x} \mathcal{E}_{x}^{2}\right)+\omega\left(f_{1}, f_{2}\right)\left(m_{y} \mathcal{E}_{y, y^{\prime}} \oplus m_{y^{\prime}} \mathcal{E}_{y, y^{\prime}}^{2}\right)
$$

and

$$
m_{x} \mathcal{E}_{x}^{3} \subset t f\left(m_{x} \mathcal{E}_{x}^{2}\right)+\left(f_{1}, f_{2}\right)^{*} m_{y, y^{\prime}} \mathcal{E}_{x}^{3} .
$$

Thus, by theorem (2.1), $f=\left(f_{1}, f_{2}\right)$ is 3-determined with respect to equivalence over varieties.

\section{Reduction of theorems (2.1) and (2.2)}

Theorem (2.1) (resp. theorem (2.2)) derives from the following theorem (3.1) (resp. theorem (3.2)).

Theorem 3.1: Let $f=\left(f_{1}, f_{2}\right), g=\left(g_{1}, g_{2}\right):\left(\mathbb{R}^{n}, 0\right) \rightarrow\left(\mathbb{R}^{p} \times \mathbb{R}^{q},(0,0)\right)$ be two $C^{\infty}$ divergent diagrams. Suppose there exist a $C^{\infty}$ diffeomorphic map germ $s:\left(\mathbb{R}^{n}, 0\right) \rightarrow\left(\mathbb{R}^{n}, 0\right)$ and a $C^{\infty}$ map germ

$$
M(x)=\left(\mathbf{m}_{1}(x), \ldots, \mathbf{m}_{p+q}(x)\right):\left(\mathbb{R}^{n}, 0\right) \rightarrow\left(G L(p+q, \mathbb{R}), E_{p+q}\right)
$$

such that $f(x)=M(x)(g \circ s)(x)$. Suppose furthermore there exists a positive integer $k$ such that

$$
\mathbf{m}_{i}(x)-\mathbf{m}_{i}(0) \in m_{x}^{k} \mathcal{E}_{x}^{p+q} \subset t f\left(m_{x} \mathcal{E}_{x}^{n}\right)+\omega\left(f_{1}, f_{2}\right)\left(m_{y} \mathcal{E}_{y, y^{\prime}}^{p} \oplus m_{y^{\prime}} \mathcal{E}_{y, y^{\prime}}^{q}\right)
$$

for any $i \quad(1 \leq i \leq p+q)$. Then $f$ and $g$ are equivalent over varieties.
Theorem 3.2: Let $f=\left(f_{1}, f_{2}\right), g=\left(g_{1}, g_{2}\right):\left(\mathbb{R}^{n}, 0\right) \rightarrow\left(\mathbb{R}^{p} \times \mathbb{R}^{q},(0,0)\right)$ be two $C^{\infty}$ divergent diagrams. Suppose there exist a $C^{\infty}$ diffeomorphic map germ $s:\left(\mathbb{R}^{n}, 0\right) \rightarrow\left(\mathbb{R}^{n}, 0\right)$ and $a C^{\infty}$ map germ

$$
N(x)=\left(\mathbf{n}_{1}(x), \ldots, \mathbf{n}_{p+q}(x)\right):\left(\mathbb{R}^{n}, 0\right) \rightarrow\left(G L(p+q, \mathbb{R}), E_{p+q}\right)
$$

such that the following two properties hold:

$$
f(x)=N(x)(g \circ s)(x)
$$

$$
\mathbf{n}_{i}(x) \text { is the constant vector } \mathbf{e}_{i} \quad \text { for any } i \quad(1 \leq i \leq p) .
$$

Suppose furthermore there exists a positive integer $k$ such that

$$
\mathbf{n}_{i}(x)-\mathbf{n}_{i}(0) \in m_{x}^{k} \mathcal{E}_{x}^{p+q} \subset t f\left(m_{x} \mathcal{E}_{x}^{n}\right)+\omega f_{2}\left(m_{y^{\prime}} \mathcal{E}_{y^{\prime}}^{p+q}\right)
$$

for any $i \quad(1 \leq i \leq p+q)$. Then $f$ and $g$ are $\mathcal{R}^{+}$-equivalent.

Proof that Theorem (3.1) implies Theorem (2.1): Let ${ }_{i} \mathcal{K}(n ; p, q)$ be the set of all pairs of $(s, M)$, where $s:\left(\mathbb{R}^{n}, 0\right) \rightarrow\left(\mathbb{R}^{n}, 0\right)$ is a $C^{\infty}$ diffeomorphic map germ and $M(x)=\left(\mathbf{m}_{1}(x), \ldots, \mathbf{m}_{p+q}(x)\right):\left(\mathbb{R}^{n}, 0\right) \rightarrow\left(G L(p+q, \mathbb{R}), E_{p+q}\right)$ is a $C^{\infty}$ map germ with the following property.

$$
\text { each element of } M(x)-M(0) \text { is included in } m_{x}^{i+1} \mathcal{E}_{x} \text {. }
$$

The set ${ }_{i} \mathcal{K}(n ; p, q)$ becomes a group by the operation $\left(s_{1}, M_{1}\right) *\left(s_{2}, M_{2}\right)=$ $\left(s_{1} \circ s_{2}, M_{1} M_{2}\right)$, where $s_{1} \circ s_{2}$ means the composition of $s_{1}$ and $s_{2}$ and $M_{1} M_{2}$ means the product of matrices $M_{1}$ and $M_{2}$. In fact, we may consider that ${ }_{i} \mathcal{K}(n ; p, q)$ is a subgroup of the group $\mathcal{K}$ introduced by J. Mather in [M1].

We want to study the orbit through a given $C^{\infty}$ divergent diagram $f=$ $\left(f_{1}, f_{2}\right):\left(\mathbb{R}^{n}, 0\right) \rightarrow\left(\mathbb{R}^{p} \times \mathbb{R}^{q},(0,0)\right)$ by the action of the group ${ }_{i} \mathcal{K}(n ; p, q)$. The tangent space at $f=\left(f_{1}, f_{2}\right)$ to this orbit is

$$
t f\left(m_{x} \mathcal{E}_{x}^{p}\right)+\left(f_{1}, f_{2}\right)^{*} m_{y, y^{\prime}} m_{x}^{i+1} \mathcal{E}_{x}^{p+q} .
$$


Since (3.3) is an $\mathcal{E}_{x}$-module, by an ordinary argument (c.f. [W]) we may prove easily

Lemma 3.4: Let $f=\left(f_{1}, f_{2}\right):\left(\mathbb{R}^{n}, 0\right) \rightarrow\left(\mathbb{R}^{p} \times \mathbb{R}^{q},(0,0)\right)$ be a $C^{\infty}$ divergent diagram. Suppose there exist positive integers $k, \ell$ such that

$$
m_{x}^{\ell} \mathcal{E}_{x}^{p} \subset t f\left(m_{x} \mathcal{E}_{x}^{n}\right)+\left(f_{1}, f_{2}\right)^{*} m_{y, y^{\prime}} m_{x}^{k} \mathcal{E}_{x}^{p+q} .
$$

Then $f$ is $\ell$-determined with respect to the group $_{k-1} \mathcal{K}(n ; p, q)$.

Thus, by using of Mather's lemma (lemma (3.1) of [M2]), we see

Lemma 3.5: Let $f=\left(f_{1}, f_{2}\right):\left(\mathbb{R}^{n}, 0\right) \rightarrow\left(\mathbb{R}^{p} \times \mathbb{R}^{q},(0,0)\right)$ be a $C^{\infty}$ divergent diagram. Suppose there exist positive integers $k, \ell$ such that

$$
m_{x}^{\ell} \mathcal{E}_{x}^{p} \subset t f\left(m_{x} \mathcal{E}_{x}^{n}\right)+\left(f_{1}, f_{2}\right)^{*} m_{y, y^{\prime}} m_{x}^{k} \mathcal{E}_{x}^{p+q} .
$$

Then $f$ is $(\ell-1)$-determined with respect to the group $_{k-1} \mathcal{K}(n ; p, q)$ if, and only if, for any $C^{\infty}$ divergent diagram $g=\left(g_{1}, g_{2}\right):\left(\mathbb{R}^{n}, 0\right) \rightarrow\left(\mathbb{R}^{p} \times \mathbb{R}^{q},(0,0)\right)$ with $j^{\ell-1} g(0)=j^{\ell-1} f(0)$, the condition

$$
m_{x}^{\ell} \mathcal{E}_{x}^{p+q} \subset \operatorname{tg}\left(m_{x} \mathcal{E}_{x}^{n}\right)+\left(g_{1}, g_{2}\right)^{*} m_{y, y^{\prime}} m_{x}^{k} \mathcal{E}_{x}^{p+q}+m_{x}^{\ell+1} \mathcal{E}_{x}^{p+q}
$$

satisfies.

Now, we start to prove that theorem (3.1) implies theorem (2.1). By the second condition in $(2.1)$, we have

$$
m_{x}^{k+\ell} \mathcal{E}_{x}^{p+q} \subset t f\left(m_{x}^{2} \mathcal{E}_{x}^{n}\right)+\left(f_{1}, f_{2}\right)^{*} m_{y, y^{\prime}} m_{x}^{k} \mathcal{E}_{x}^{p+q}
$$

Let $g=\left(g_{1}, g_{2}\right):\left(\mathbb{R}^{n}, 0\right) \rightarrow\left(\mathbb{R}^{p} \times \mathbb{R}^{q},(0,0)\right)$ be a $C^{\infty}$ divergent diagram with $j^{\ell-1} g(0)=j^{\ell-1} f(0)$. Then, since the difference $(g-f)$ is included in $m_{x}^{l} \mathcal{E}_{x}^{p}$, (3.6) implies

$$
m_{x}^{k+!} \mathcal{E}_{x}^{p+q} \subsetneq t g\left(m_{x}^{2} \mathcal{E}_{x}^{n}\right)+\left(g_{1}, g_{2}\right)^{*} m_{y, y^{\prime}} m_{x}^{k} \mathcal{E}_{x}^{p+q}+m_{x}^{\ell+1} \mathcal{E}_{x}^{p+q}
$$

Hence, by lemma (3.5), $f$ is $(k+\ell-1)$-determined with respect to the group ${ }_{k-1} \mathcal{K}(n ; p, q)$. Thus, for any $C^{\infty}$ divergent diagram $g=\left(g_{1}, g_{2}\right):\left(\mathbb{R}^{n}, 0\right) \rightarrow$
$\left(\mathbb{R}^{p} \times \mathbb{R}^{q},(0,0)\right)$ with $j^{k+\ell-1} g(0)=j^{k+\ell-1} f(0)$, there exist a $C^{\infty}$ diffeomorphic map germ $s:\left(\mathbb{R}^{n}, 0\right) \rightarrow\left(\mathbb{R}^{n}, 0\right)$ and a $C^{\infty}$ map germ

$$
M(x)=\left(\mathbf{m}_{1}(x), \ldots, \mathbf{m}_{p+q}(x)\right):\left(\mathbb{R}^{n}, 0\right) \rightarrow\left(G L(p+q, \mathbb{R}), E_{p+q}\right)
$$

such that the following two properties hold:

$$
\begin{aligned}
& f(x)=M(x)(g \circ s)(x), \\
& \mathbf{m}_{i}(x)-\mathbf{m}_{i}(0) \in m_{x}^{k} \mathcal{E}_{x}^{p+q} \quad \text { for any } i \quad(1 \leq i \leq p+q) .
\end{aligned}
$$

Therefore, by the first condition of theorem (2.1), theorem (3.1) induces that $f$ and $g$ are equivalent over varieties.

Proof that Theorem (3.2) implies theorem (2.2): First, we need several preparations.

Let ${ }_{i} \tilde{\mathcal{K}}(n ; p, q)$ be the set of all pairs of $(s, N)$, where $s:\left(\mathbb{R}^{n}, 0\right) \rightarrow\left(\mathbb{R}^{n}, 0\right)$ is a $C^{\infty}$ diffeomorphic map germ and $N(x)=\left(\mathbf{n}_{1}(x), \ldots, \mathbf{n}_{p+q}(x)\right):\left(\mathbb{R}^{n}, 0\right) \rightarrow$ $\left(G L(p+q, \mathbb{R}), E_{p+q}\right)$ is a $C^{\infty}$ map germ with the following two properties.

$$
\text { each element of } N(x)-N(0) \text { is included in } m_{x}^{i+1} \mathcal{E}_{x} \text {, }
$$

$\mathbf{n}_{i}(x)$ is the constant vector $\mathbf{e}_{i}$ for any $i \quad(1 \leq i \leq p)$.

The set ${ }_{i} \tilde{\mathcal{K}}(n ; p, q)$ becomes a group by the operation $\left(s_{1}, N_{1}\right) *\left(s_{2}, N_{2}\right)=\left(s_{1}\right.$ 。 $\left.s_{2}, N_{1} N_{2}\right)$, where $s_{1} \circ s_{2}$ means the composition of $s_{1}$ and $s_{2}$ and $N_{1} N_{2}$ means the product of matrices $N_{1}$ and $N_{2}$. In fact, we may consider that ${ }_{i} \tilde{\mathcal{K}}(n ; p, q)$ is a subgroup of the group $\mathcal{K}$ introduced by J. Mather in [M1].

We want to study the orbit through a given $C^{\infty}$ divergent diagram $f=$ $\left(f_{1}, f_{2}\right):\left(\mathbb{R}^{n}, 0\right) \rightarrow\left(\mathbb{R}^{p} \times \mathbb{R}^{q},(0,0)\right)$ by the action of the group ${ }_{i} \tilde{\mathcal{K}}(n ; p, q)$. The tangent space at $f=\left(f_{1}, f_{2}\right)$ to this orbit is

$$
t f\left(m_{x} \mathcal{E}_{x}^{n}\right)+f_{2}^{*} m_{y^{\prime}} m_{x}^{i+1} \mathcal{E}_{x}^{p+q}
$$

Since (3.7) is an $\mathcal{E}_{x}$-module, by an ordinary argument (c.f. [W]) we may prove easily 
Lemma 3.8: Let $f=\left(f_{1}, f_{2}\right):\left(\mathbb{R}^{n}, 0\right) \rightarrow\left(\mathbb{R}^{p} \times \mathbb{R}^{q},(0,0)\right)$ be a $C^{\infty}$ divergent diagram. Suppose there exist positive integers $k, \ell$ such that

$$
m_{x}^{\ell} \mathcal{E}_{x}^{p} \subset t f\left(m_{x} \mathcal{E}_{x}^{n}\right)+f_{2}^{*} m_{y^{\prime}} m_{x}^{k} \mathcal{E}_{x}^{p+q}
$$

Then $f$ is $\ell$-determined with respect to the group $_{k-1} \tilde{\mathcal{K}}(n ; p, q)$.

Thus, by using of Mather's lemma (lemma (3.1) of [M2]), we see

Lemma 3.9: Let $f=\left(f_{1}, f_{2}\right):\left(\mathbb{R}^{n}, 0\right) \rightarrow\left(\mathbb{R}^{p} \times \mathbb{R}^{q},(0,0)\right)$ be a $C^{\infty}$ divergent diagram. Suppose there exist positive integers $k, \ell$ such that

$$
m_{x}^{\ell} \mathcal{E}_{x}^{p} \subset t f\left(m_{x} \mathcal{E}_{x}^{n}\right)+f_{2}^{*} m_{y^{\prime}} m_{x}^{k} \mathcal{E}_{x}^{p+q} .
$$

Then $f$ is $(\ell-1)$-determined with respect to the group $_{k-1} \tilde{\mathcal{K}}(n ; p, q)$ if, and only if, for any $C^{\infty}$ divergent diagram $g=\left(g_{1}, g_{2}\right):\left(\mathbb{R}^{n}, 0\right) \rightarrow\left(\mathbb{R}^{p} \times \mathbb{R}^{q},(0,0)\right)$ with $j^{\ell-1} g(0)=j^{\ell-1} f(0)$, the condition

$$
m_{x}^{\ell} \mathcal{E}_{x}^{p+q} \subset t g\left(m_{x} \mathcal{E}_{x}^{n}\right)+g_{2}^{*} m_{y^{\prime}} m_{x}^{k} \mathcal{E}_{x}^{p+q}+m_{x}^{\ell+1} \mathcal{E}_{x}^{p+q}
$$

satisfies.

Now, we start to prove that theorem (3.2) implies theorem (2.2). By the second condition in $(2.2)$, we have

$$
m_{x}^{k+\ell} \mathcal{E}_{x}^{p+q} \subset t f\left(m_{x}^{2} \mathcal{E}_{x}^{n}\right)+f_{2}^{*} m_{y^{\prime}} m_{x}^{k} \mathcal{E}_{x}^{p+q} .
$$

Let $g=\left(g_{1}, g_{2}\right):\left(\mathbb{R}^{n}, 0\right) \rightarrow\left(\mathbb{R}^{p} \times \mathbb{R}^{q},(0,0)\right)$ be a $C^{\infty}$ divergent diagram with $j^{k+\ell-1} g(0)=j^{k+\ell-1} f(0)$. Then, since the difference $(g-f)$ is included in $m_{x}^{k+\ell} \mathcal{E}_{x}^{p+g},(3.10)$ implies

$$
m_{x}^{k+\ell} \mathcal{E}_{x}^{p+q} \subset \operatorname{tg}\left(m_{x}^{2} \mathcal{E}_{x}^{n}\right)+g_{2}^{*} m_{y^{\prime}} m_{x}^{k} \mathcal{E}_{x}^{p+q}+m_{x}^{k+\ell+1} \mathcal{E}_{x}^{p+q} .
$$

Hence, by lemma (3.9), $f$ is $(k+\ell-1)$-determined with respect to the group ${ }_{k-1} \tilde{\mathcal{K}}(n ; p, q)$. Thus, for any $C^{\infty}$ divergent diagram $g:\left(\mathbb{R}^{n}, 0\right) \rightarrow\left(\mathbb{R}^{p} \times\right.$ $\left.\mathbb{R}^{q},(0,0)\right)$ with $j^{k+\ell-1} g(0)=j^{k+\ell-1} f(0)$, there exist a $C^{\infty}$ diffeomorphic map germ $s:\left(\mathbb{R}^{n}, 0\right) \rightarrow\left(\mathbb{R}^{n}, 0\right)$ and a $C^{\infty}$ map germ

$$
N(x)=\left(\mathbf{n}_{1}(x), \ldots, \mathbf{n}_{p+q}(x)\right):\left(\mathbb{R}^{n}, 0\right) \rightarrow\left(G L(p+q, \mathbb{R}), E_{p+q}\right)
$$

such that the following three properties hold:

$$
\begin{aligned}
& f(x)=N(x)(g \circ s)(x), \\
& \mathbf{n}_{i}(x) \text { is the constant vector } \mathbf{e}_{i} \text { for any } i \quad(1 \leq i \leq p), \\
& \mathbf{n}_{i}(x)-\mathbf{n}_{i}(0) \in m_{x}^{k} \mathcal{E}_{x}^{p+q} \text { for any } i \quad(1 \leq i \leq p+q) .
\end{aligned}
$$

Therefore, by the first condition of theorem (2.2), theorem (3.2) induces that $f$ and $g$ are $\mathcal{R}^{+}$-equivalent.

\section{Algebraic Lemmata Concerning Mixed Homomor- phisms}

In this chapter, we prove two algebraic lemmata (lemma (4.2) and lemma (4.4)) concerning mixed homomorphisms in the sense of J. Mather ([M1]). We need Malgrange's preparation theorem $([\mathrm{Mg}])$ in this chapter.

Let $f=\left(f_{1}, f_{2}\right):\left(\mathbb{R}^{n}, 0\right) \rightarrow\left(\mathbb{R}^{p} \times \mathbb{R}^{q},(0,0)\right)$ be a $C^{\infty}$ divergent diagram and let

$$
M(x)=\left(\mathbf{m}_{1}(x), \ldots, \mathbf{m}_{p+q}(x)\right):\left(\mathbb{R}^{n}, 0\right) \rightarrow\left(G L(p+q, \mathbb{R}), E_{p+q}\right)
$$

be a $C^{\infty}$ map germ. Let $\Phi=\left(\Phi_{1}, \Phi_{2}\right):\left(\mathbb{R}^{n} \times \mathbb{R}_{\lambda}^{p} \times \mathbb{R}_{\lambda^{\prime}}^{q},(0,0,0)\right) \rightarrow\left(\mathbb{R}_{y}^{p} \times\right.$ $\left.\mathbb{R}_{y^{\prime}}^{q},(0,0)\right)$ be the $C^{\infty}$ deformation of $f$ having the following form:

$$
\left[\begin{array}{l}
\Phi_{1}\left(x, \lambda, \lambda^{\prime}\right) \\
\Phi_{2}\left(x, \lambda, \lambda^{\prime}\right)
\end{array}\right]=\left[\begin{array}{l}
f_{1}(x) \\
f_{2}(x)
\end{array}\right]-M(x)\left[\begin{array}{c}
\lambda \\
\lambda^{\prime}
\end{array}\right]
$$

Lemma 4.2: Suppose there exists a positive integer $k$ such that

$$
\mathbf{m}_{i}(x)-\mathbf{m}_{i}(0) \in m_{x}^{k} \mathcal{E}_{x}^{p+q} \subset t f\left(m_{x} \mathcal{E}_{x}^{n}\right)+\omega\left(f_{1}, f_{2}\right)\left(m_{y} \mathcal{E}_{y, y^{\prime}}^{p} \oplus m_{y^{\prime}} \mathcal{E}_{y, y^{\prime}}^{q}\right)
$$

for any $i \quad(1 \leq i \leq p+q)$. Then $\mathbf{m}_{i}(x)-\mathbf{m}_{i}(0)$ is included in

$$
t \Phi_{x}\left(m_{x} \mathcal{E}_{x, \lambda, \lambda^{\prime}}^{n}\right)+\omega\left(\Phi_{1}, \Phi_{2}, \pi_{\lambda}, \pi_{\lambda^{\prime}}\right)\left(m_{y, \lambda} \mathcal{E}_{y, y^{\prime}, \lambda, \lambda^{\prime}}^{p} \oplus m_{y^{\prime}, \lambda^{\prime}} \mathcal{E}_{y, y^{\prime}, \lambda, \lambda^{\prime}}^{q}\right)
$$

for any $i \quad(1 \leq i \leq p+q)$.

Among deformations of type (4.1), we give the following deformations (type (4.3)) special treatment. Let

$$
N(x)=\left(\mathbf{n}_{1}(x), \ldots, \mathbf{n}_{p+q}(x)\right):\left(\mathbb{R}^{n}, 0\right) \rightarrow\left(G L(p+q, \mathbb{R}), E_{p+q}\right)
$$


be a $C^{\infty}$ map germ such that $\mathbf{n}_{i}(x)$ is a constant vector $\mathbf{e}_{i}$ for any $i \quad(1 \leq i \leq$ $p)$. Let $\Psi=\left(\Psi_{1}, \Psi_{2}\right):\left(\mathbb{R}^{n} \times \mathbb{R}_{\lambda}^{p} \times \mathbb{R}_{\lambda^{\prime}}^{q},(0,0,0)\right) \rightarrow\left(\mathbb{R}_{y}^{p} \times \mathbb{R}_{y^{\prime}}^{q},(0,0)\right)$ be the $C^{\infty}$ deformation of $f$ having the following form:

$$
\begin{aligned}
{\left[\begin{array}{l}
\Psi_{1}\left(x, \lambda, \lambda^{\prime}\right) \\
\Psi_{2}\left(x, \lambda, \lambda^{\prime}\right)
\end{array}\right] } & =\left[\begin{array}{c}
f_{1}(x) \\
f_{2}(x)
\end{array}\right]-N(x)\left[\begin{array}{c}
\lambda \\
\lambda^{\prime}
\end{array}\right] \\
& =\left[\begin{array}{c}
f_{1}(x)-\lambda-N_{1}(x) \lambda^{\prime} \\
f_{2}(x)-N_{2}(x) \lambda^{\prime}
\end{array}\right],
\end{aligned}
$$

where we set

$$
N(x)=\left[\begin{array}{cc}
E_{p} & N_{1}(x) \\
O & N_{2}(x)
\end{array}\right]
$$

Lemma 4.4: Suppose there exists a positive integer $k$ such that

$$
\mathbf{n}_{i}(x)-\mathbf{n}_{i}(0) \in m_{x}^{k} \mathcal{E}_{x}^{p+q} \subset t f\left(m_{x} \mathcal{E}_{x}^{n}\right)+\omega f_{2}\left(m_{y^{\prime}} \mathcal{E}_{y^{\prime}}^{p+q}\right)
$$

for any $i \quad(1 \leq i \leq p+q)$. Then $\mathbf{n}_{i}(x)-\mathbf{n}_{i}(0)$ is included in

$$
t \Psi_{x}\left(m_{x} \mathcal{E}_{x, \lambda^{\prime}}^{n}\right)+\omega\left(\Psi_{2}, \pi_{\lambda^{\prime}}\right)\left(m_{y^{\prime}, \lambda^{\prime}} \mathcal{E}_{y^{\prime}, \lambda^{\prime}}^{p+q}\right)
$$

for any $i \quad(1 \leq i \leq p+q)$.

Proof of Lemma (4.2): Since we assumed

$$
m_{x}^{k} \mathcal{E}_{x}^{p+q} \subset t f\left(m_{x} \mathcal{E}_{x}^{n}\right)+\omega\left(f_{1}, f_{2}\right)\left(m_{y} \mathcal{E}_{y, y^{\prime}}^{p} \oplus m_{y^{\prime}} \mathcal{E}_{y, y^{\prime}}^{q}\right)
$$

by Malgrange's preparation theorem $([\mathrm{Mg}])$ we have

$$
\text { (4.5) } \begin{aligned}
m_{x}^{k} \mathcal{E}_{x, \lambda, \lambda^{\prime}}^{p+q} & \subset t f\left(m_{x} \mathcal{E}_{x, \lambda, \lambda^{\prime}}^{n}\right) \\
& +\omega\left(f_{1}, f_{2}, \pi_{\lambda}, \pi_{\lambda^{\prime}}\right)\left(m_{y} \mathcal{E}_{y, y^{\prime}, \lambda, \lambda^{\prime}}^{p} \oplus m_{y^{\prime}} \mathcal{E}_{y, y^{\prime}, \lambda, \lambda^{\prime}}^{q}\right) .
\end{aligned}
$$

We set $\tilde{\Phi}=\left(\tilde{\Phi}_{1}, \tilde{\Phi}_{2}\right):\left(\mathbb{R}^{n} \times \mathbb{R}_{\lambda}^{p} \times \mathbb{R}_{\lambda^{\prime}}^{q},(0,0,0)\right) \rightarrow\left(\mathbb{R}_{y}^{p} \times \mathbb{R}_{y^{\prime}}^{q},(0,0)\right)$ as

$$
\begin{aligned}
{\left[\begin{array}{c}
\tilde{\Phi}_{1}\left(x, \lambda, \lambda^{\prime}\right) \\
\tilde{\Phi}_{2}\left(x, \lambda, \lambda^{\prime}\right)
\end{array}\right] } & =\left[\begin{array}{c}
\Phi_{1}\left(x, \lambda, \lambda^{\prime}\right) \\
\Phi_{2}\left(x, \lambda, \lambda^{\prime}\right)
\end{array}\right]+\left[\begin{array}{cc}
E_{p} & O \\
O & E_{q}
\end{array}\right]\left[\begin{array}{c}
\lambda \\
\lambda^{\prime}
\end{array}\right] \\
& =\left[\begin{array}{c}
f_{1}(x) \\
f_{2}(x)
\end{array}\right]-(M(x)-M(0))\left[\begin{array}{c}
\lambda \\
\lambda^{\prime}
\end{array}\right]
\end{aligned}
$$

Since we assumed

$$
\mathbf{m}_{i}(x)-\mathbf{m}_{i}(0) \in m_{x}^{k} \mathcal{E}_{x}^{p+q}
$$

for any $i(1 \leq i \leq p)$, the difference

$$
\begin{aligned}
\tilde{\Phi}\left(x, \lambda, \lambda^{\prime}\right)-f(x) & =(M(x)-M(0))\left[\begin{array}{c}
\lambda \\
\lambda^{\prime}
\end{array}\right] \\
& =-\sum_{i=1}^{p} \lambda_{i}\left(\mathbf{m}_{i}(x)-\mathbf{m}_{i}(0)\right)-\sum_{i=1}^{q} \lambda_{i}^{\prime}\left(\mathbf{m}_{p+i}(x)-\mathbf{m}_{p+i}(0)\right)
\end{aligned}
$$

is included in

$$
\left(\pi_{\lambda}, \pi_{\lambda^{\prime}}\right)^{*} m_{\lambda, \lambda^{\prime}} m_{x}^{k} \mathcal{E}_{x, \lambda, \lambda^{\prime}}^{p+q}
$$

$\subset\left(\tilde{\Phi}_{1}, \tilde{\Phi}_{2}, \pi_{\lambda}, \pi_{\lambda^{\prime}}\right)^{*} m_{y, y^{\prime}, \lambda, \lambda^{\prime}} m_{x}^{k} \mathcal{E}_{x, \lambda, \lambda^{\prime}}^{p+q}$.

Hence, we can approximate (4.5) as follows.

$$
\begin{aligned}
m_{x}^{k} \mathcal{E}_{x, \lambda, \lambda^{\prime}}^{p+q} & \subset t \tilde{\Phi}_{x}\left(m_{x} \mathcal{E}_{x, \lambda, \lambda^{\prime}}^{n}\right) \\
& +\omega\left(\tilde{\Phi}_{1}, \tilde{\Phi}_{2}, \pi_{\lambda}, \pi_{\lambda^{\prime}}\right)\left(m_{y} \mathcal{E}_{y, y^{\prime}, \lambda, \lambda^{\prime}}^{p} \oplus m_{y^{\prime}} \mathcal{E}_{y, y^{\prime}, \lambda, \lambda^{\prime}}^{q}\right) \\
& +\left(\tilde{\Phi}_{1}, \tilde{\Phi}_{2}, \pi_{\lambda}, \pi_{\lambda^{\prime}}\right)^{*} m_{y, y^{\prime}, \lambda, \lambda^{\prime}} m_{x}^{k} \mathcal{E}_{x, \lambda, \lambda^{\prime}}^{p+q}
\end{aligned}
$$

We set

$$
\begin{aligned}
C= & \mathcal{E}_{x, \lambda, \lambda^{\prime}}^{p+q} / t \tilde{\Phi}_{x}\left(m_{x} \mathcal{E}_{x, \lambda, \lambda^{\prime}}^{n}\right) \\
A= & \text { image of } \omega\left(\widetilde{\Phi}_{1}, \widetilde{\Phi}_{2}, \pi_{\lambda}, \pi_{\lambda^{\prime}}\right)\left(m_{y} \mathcal{E}_{y, y^{\prime}, \lambda, \lambda^{\prime}}^{p} \oplus m_{y^{\prime}} \mathcal{E}_{y, y^{\prime}, \lambda, \lambda^{\prime}}^{q}\right) \\
& \text { by the canonical projection to } C, \\
B= & m_{x}^{k} \cdot C .
\end{aligned}
$$

Then, by (4.6) we have

$$
B \subset A+\left(\tilde{\Phi}_{1}, \tilde{\Phi}_{2}, \pi_{\lambda}, \pi_{\lambda^{\prime}}\right)^{*} m_{y, y^{\prime}, \lambda, \lambda^{\prime}} B
$$

Since

$$
\begin{aligned}
& \operatorname{dim}_{\mathbb{R}} B /\left(\widetilde{\Phi}_{1}, \tilde{\Phi}_{2}, \pi_{\lambda}, \pi_{\lambda^{\prime}}\right)^{*} m_{y, y^{\prime}, \lambda, \lambda^{\prime}} B \\
= & \operatorname{dim}_{\mathbb{R}^{R}} m_{x}^{k} \mathcal{E}_{x}^{p+q} / m_{x}^{k}\left(t f\left(m_{x} \mathcal{E}_{x}^{n}\right)+\left(f_{1}, f_{2}\right)^{*} m_{y, y^{\prime}} \mathcal{E}_{x}^{p+q}\right)<\infty,
\end{aligned}
$$

by Malgrange's preparation theorem $([\mathrm{Mg}])$ we see that $B$ is finitely generated $\mathcal{E}_{y, y^{\prime}, \lambda, \lambda^{\prime}}$-module via $\left(\tilde{\Phi}_{1}, \tilde{\Phi}_{2}, \pi_{\lambda}, \pi_{\lambda^{\prime}}\right)$. Hence, by Nakayama's lemma (4.7) implies

$$
B \subset A \text {. }
$$

From the form

$$
\left[\begin{array}{c}
\tilde{\Phi}_{1}\left(x, \lambda, \lambda^{\prime}\right) \\
\tilde{\Phi}_{2}\left(x, \lambda, \lambda^{\prime}\right)
\end{array}\right]=\left[\begin{array}{c}
\Phi_{1}\left(x, \lambda, \lambda^{\prime}\right) \\
\Phi_{2}\left(x, \lambda, \lambda^{\prime}\right)
\end{array}\right]+\left[\begin{array}{cc}
E_{p} & O \\
O & E_{q}
\end{array}\right]\left[\begin{array}{c}
\lambda \\
\lambda^{\prime}
\end{array}\right],
$$

we see 
(4.9) $\quad t \tilde{\Phi}_{x}\left(m_{x} \mathcal{E}_{x, \lambda, \lambda^{\prime}}^{n}\right)+\omega\left(\tilde{\Phi}_{1}, \widetilde{\Phi}_{2}, \pi_{\lambda}, \pi_{\lambda^{\prime}}\right)\left(m_{y} \mathcal{E}_{y, y^{\prime}, \lambda, \lambda^{\prime}}^{p} \oplus m_{y^{\prime}} \mathcal{E}_{y, y^{\prime}, \lambda, \lambda^{\prime}}^{q}\right)$ $\subset t \Phi_{x}\left(m_{x} \mathcal{E}_{x, \lambda, \lambda^{\prime}}^{n}\right)+\omega\left(\Phi_{1}, \Phi_{2}, \pi_{\lambda}, \pi_{\lambda^{\prime}}\right)\left(m_{y, \lambda} \mathcal{E}_{y, y^{\prime}, \lambda, \lambda^{\prime}}^{p} \oplus m_{y^{\prime}, \lambda^{\prime}} \mathcal{E}_{y, y^{\prime}, \lambda, \lambda^{\prime}}^{q}\right)$.

(4.8) and (4.9) yields

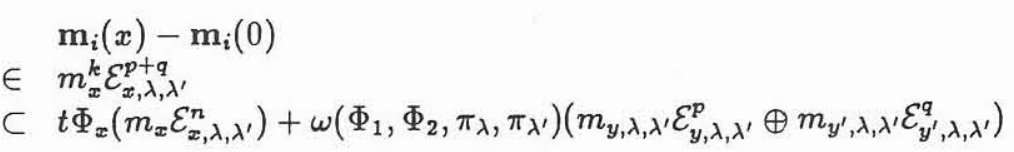

for any $i \quad(1 \leq i \leq p+q)$. $\quad \square$

Proof of Lemma (4.4): Since we assumed

$$
m_{x}^{k} \mathcal{E}_{x}^{p+q} \subset t f\left(m_{x} \mathcal{E}_{x}^{n}\right)+\omega f_{2}\left(m_{y^{\prime}} \mathcal{E}_{y^{\prime}}^{p+q}\right),
$$

by Malgrange's preparation theorem $([\mathrm{Mg}])$ we have

$$
m_{x}^{k} \mathcal{E}_{x, \lambda^{\prime}}^{p+q} \subset t f\left(m_{x} \mathcal{E}_{x, \lambda^{\prime}}^{n}\right)+\omega\left(f_{2}, \pi_{\lambda^{\prime}}\right)\left(m_{y^{\prime}} \mathcal{E}_{y^{\prime}, \lambda^{\prime}}^{p+q}\right) .
$$

We set

$$
\begin{aligned}
\tilde{\Psi}=\left(\tilde{\Psi}_{1}, \tilde{\Psi}_{2}\right):\left(\mathbb{R}^{n} \times \mathbb{R}_{\lambda^{\prime}}^{q},(0,0,0)\right) \rightarrow\left(\mathbb{R}_{y}^{p} \times \mathbb{R}_{y^{\prime}}^{q},(0,0)\right) & \text { as } \\
{\left[\begin{array}{c}
\tilde{\Psi}_{1}\left(x, \lambda^{\prime}\right) \\
\tilde{\Psi}_{2}\left(x, \lambda^{\prime}\right)
\end{array}\right] } & =\left[\begin{array}{c}
\Psi_{1}\left(x, \lambda, \lambda^{\prime}\right) \\
\Psi_{2}\left(x, \lambda, \lambda^{\prime}\right)
\end{array}\right]+N(0)\left[\begin{array}{c}
\lambda \\
\lambda^{\prime}
\end{array}\right] \\
& =\left[\begin{array}{c}
f_{1}(x) \\
f_{2}(x)
\end{array}\right]-(N(x)-N(0))\left[\begin{array}{c}
0 \\
\lambda^{\prime}
\end{array}\right] .
\end{aligned}
$$

Since we assumed

$$
\mathbf{n}_{i}(x)-\mathbf{n}_{i}(0) \in m_{x}^{k} \mathcal{E}_{x}^{p+q}
$$

for any $i(1 \leq i \leq p+q)$, the difference

$$
\begin{aligned}
\tilde{\Psi}\left(x, \lambda^{\prime}\right)-f(x) & =(N(x)-N(0))\left[\begin{array}{c}
0 \\
\lambda^{\prime}
\end{array}\right] \\
& =-\sum_{i=1}^{q} \lambda_{i}^{\prime}\left(\mathbf{n}_{p+i}(x)-\mathbf{n}_{p+i}(0)\right)
\end{aligned}
$$

is included in

$$
\subset \quad\left(\begin{array}{l}
\pi_{\lambda}^{*} m_{\lambda^{\prime}} m_{x}^{k} \mathcal{E}_{x, \lambda^{\prime}}^{p+q} \\
\left.\tilde{\Psi}_{2}, \pi_{\lambda^{\prime}}\right)^{*} m_{y, y^{\prime}, \lambda^{\prime}} m_{x}^{k} \mathcal{E}_{x, \lambda^{\prime}}^{p+q} .
\end{array}\right.
$$

Hence, we can approximate (4.10) as follows.

$$
\begin{aligned}
m_{x}^{k} \mathcal{E}_{x, \lambda^{\prime}}^{p+q} \subset t \tilde{\Psi}_{x}\left(m_{x} \mathcal{E}_{x, \lambda^{\prime}}^{n}\right)+\omega\left(\tilde{\Psi}_{2}, \pi_{\lambda^{\prime}}\right)\left(m_{y^{\prime}} \mathcal{E}_{y^{\prime}, \lambda^{\prime}}^{p+q}\right) \\
+\left(\tilde{\Psi}_{1}, \tilde{\Psi}_{2}, \pi_{\lambda^{\prime}}\right)^{*} m_{y, y^{\prime}, \lambda^{\prime}} m_{x}^{k} \mathcal{E}_{x, \lambda^{\prime}}^{p+q}
\end{aligned}
$$

We set

$$
\begin{aligned}
C= & \mathcal{E}_{x, \lambda^{\prime}}^{p+q} / t \tilde{\Psi}_{x}\left(m_{x} \mathcal{E}_{x, \lambda^{\prime}}^{n}\right), \\
A= & \text { image of } \omega\left(\tilde{\Psi}_{2}, \pi_{\lambda^{\prime}}\right)\left(m_{y^{\prime}} \mathcal{E}_{y^{\prime}, \lambda^{\prime}}^{p+q}\right) \\
& \text { by the canonical projection to } C, \\
B= & m_{x}^{k} . C .
\end{aligned}
$$

Then, by (4.11) we have

$$
B \subset A+\left(\tilde{\Psi}_{1}, \tilde{\Psi}_{2}, \pi_{\lambda^{\prime}}\right)^{*} m_{y, y^{\prime}, \lambda^{\prime}} B .
$$

Since

$$
\begin{aligned}
& \operatorname{dim}_{\mathbb{R}} B /\left(\tilde{\Psi}_{1}, \tilde{\Psi}_{2}, \pi_{\lambda^{\prime}}\right)^{*} m_{y, y^{\prime}, \lambda^{\prime}} B \\
= & \operatorname{dim}_{\mathbb{R}} m_{x}^{k} \mathcal{E}_{x}^{p+q} / m_{x}^{k}\left(t f\left(m_{x} \mathcal{E}_{x}^{n}\right)+\left(f_{1}, f_{2}\right)^{*} m_{y, y^{\prime}} \mathcal{E}_{x}^{p+q}\right)<\infty,
\end{aligned}
$$

by Malgrange's preparation theorem $([\mathrm{Mg}])$ we see that $B$ is finitely generated $\mathcal{E}_{y, y^{\prime}, \lambda^{\prime}}$-module via $\left(\tilde{\Psi}_{1}, \tilde{\Psi}_{2}, \pi_{\lambda^{\prime}}\right)$. Hence, by Nakayama's lemma (4.12) implies

$$
B \subset A \text {. }
$$

From the form

$$
\left[\begin{array}{c}
\tilde{\Psi}_{1}\left(x, \lambda^{\prime}\right) \\
\tilde{\Psi}_{2}\left(x, \lambda^{\prime}\right)
\end{array}\right]=\left[\begin{array}{l}
\Psi_{1}\left(x, \lambda, \lambda^{\prime}\right) \\
\Psi_{2}\left(x, \lambda, \lambda^{\prime}\right)
\end{array}\right]+\left[\begin{array}{cc}
E_{p} & N_{1}(0) \\
O & N_{2}(0)
\end{array}\right]\left[\begin{array}{c}
\lambda \\
\lambda^{\prime}
\end{array}\right],
$$

we see

$$
t \tilde{\Psi}_{x}\left(m_{x} \mathcal{E}_{x, \lambda^{\prime}}^{n}\right)+\omega\left(\tilde{\Psi}_{2}, \pi_{\lambda^{\prime}}\right)\left(m_{y^{\prime}} \mathcal{E}_{y^{\prime}, \lambda^{\prime}}^{p+q}\right)
$$

$$
\subset t \Psi_{x}\left(m_{x} \mathcal{E}_{x, \lambda^{\prime}}^{n}\right)+\omega\left(\Psi_{2}, \pi_{\lambda}, \pi_{\lambda^{\prime}}\right)\left(m_{y^{\prime}, \lambda^{\prime}} \mathcal{E}_{y^{\prime}, \lambda^{\prime}}^{p+q}\right) .
$$

(4.13) and (4.14) yields

$$
\begin{aligned}
& \mathbf{n}_{i}(x)-\mathbf{n}_{i}(0) \\
\in & m_{x}^{k} \mathcal{E}_{x, \lambda^{\prime}}^{p+q} \\
\subset & t \Psi_{x}\left(m_{x} \mathcal{E}_{x, \lambda^{\prime}}^{n}\right)+\omega\left(\Psi_{2}, \pi_{\lambda^{\prime}}\right)\left(m_{y^{\prime}, \lambda^{\prime}} \mathcal{E}_{y^{\prime}, \lambda^{\prime}}^{p+q}\right)
\end{aligned}
$$

for any $i \quad(1 \leq i \leq p+q)$.

\section{Proof of Theorem (3.1)}

Let $\Phi=\left(\Phi_{1}, \Phi_{2}\right):\left(\mathbb{R}^{n} \times \mathbb{R}_{\lambda}^{p} \times \mathbb{R}_{\lambda^{\prime}}^{q},(0,0,0)\right) \rightarrow\left(\mathbb{R}_{y}^{p} \times \mathbb{R}_{y^{\prime}}^{q},(0,0)\right)$ be the $C^{\infty}$ deformation of $f$ having the following form:

$$
\left[\begin{array}{l}
\Phi_{1}\left(x, \lambda, \lambda^{\prime}\right) \\
\Phi_{2}\left(x, \lambda, \lambda^{\prime}\right)
\end{array}\right]=\left[\begin{array}{c}
f_{1}(x) \\
f_{2}(x)
\end{array}\right]-M(x)\left[\begin{array}{c}
\lambda \\
\lambda^{\prime}
\end{array}\right]
$$


Since

$$
\frac{\partial \Phi}{\partial \lambda_{i}}=-\mathbf{m}_{i}(x) \text { and } \frac{\partial \Phi}{\partial \lambda_{j}^{\prime}}=-\mathbf{m}_{p+j}(x)
$$

for any $i(1 \leq i \leq p)$ and any $j(1 \leq j \leq q)$, by lemma (4.2) we can choose germs of $C^{\infty}$ vector fields

$$
\xi_{i}, \xi_{j}^{\prime} \quad \in m_{x} \mathcal{E}_{x, \lambda, \lambda^{\prime}}^{n} \text { and }
$$

$$
\eta_{i}=\left[\begin{array}{c}
\eta_{i 1} \\
\eta_{i 2}
\end{array}\right], \eta_{j}^{\prime}=\left[\begin{array}{c}
\eta_{j 1}^{\prime} \\
\eta_{j 2}^{\prime}
\end{array}\right] \in \mathcal{E}_{y, y^{\prime}, \lambda, \lambda^{\prime}}^{p+q}
$$

such that

$$
\begin{gathered}
-\frac{\partial \Phi}{\partial \lambda_{i}}=\xi_{i}(\Phi)-\eta_{i} \circ\left(\Phi_{1}, \Phi_{2}, \pi_{\lambda}, \pi_{\lambda^{\prime}}\right), \\
-\frac{\partial \Phi}{\partial \lambda_{j}^{\prime}}=\xi_{j}^{\prime}(\Phi)-\eta_{j}^{\prime} \circ\left(\Phi_{1}, \Phi_{2}, \pi_{\lambda}, \pi_{\lambda^{\prime}}\right) \\
\eta_{i}(0,0,0,0)=\frac{\partial \Phi}{\partial \lambda_{i}}(0,0,0)=\mathbf{e}_{i}, \\
\eta_{j}^{\prime}(0,0,0,0)=\frac{\partial \Phi}{\partial \lambda_{j}^{\prime}}(0,0,0)=\mathbf{e}_{p+j}, \\
\eta_{i, 2}(y, 0, \lambda, 0)=0 \\
\eta_{j, 1}^{\prime}\left(0, y^{\prime}, 0, \lambda^{\prime}\right)=0
\end{gathered}
$$

for any $i(1 \leq i \leq p)$ and any $j(1 \leq j \leq q)$.

By (5.1) and (5.2), integrating germs of $C^{\infty}$ vector fields

$$
\begin{aligned}
& \xi_{1}+\partial / \partial \lambda_{1}, \ldots, \xi_{p}+\partial / \partial \lambda_{p} \\
& \xi_{1}^{\prime}+\partial / \partial \lambda_{1}^{\prime}, \ldots, \xi_{q}^{\prime}+\partial / \partial \lambda_{q}^{\prime} \\
& \eta_{1}+\partial / \partial \lambda_{1}, \ldots, \eta_{p}+\partial / \partial \lambda_{p}
\end{aligned}
$$

and

$$
\eta_{1}^{\prime}+\partial / \partial \lambda_{1}^{\prime}, \ldots, \eta_{q}^{\prime}+\partial / \partial \lambda_{q}^{\prime}
$$

yields $C^{x}$ diffeomorphic map germs

$$
h^{-1}:\left(\mathbb{R}^{n} \times \mathbb{R}_{\lambda}^{p} \times \mathbb{R}_{\lambda^{\prime}}^{q}(0,0,0)\right) \rightarrow\left(\mathbb{R}^{n} \times \mathbb{R}_{\lambda}^{p} \times \mathbb{R}_{\lambda^{\prime}}^{q},(0,0,0)\right)
$$

\section{A CONSTRUCTIVE METHOD TO GET RIGHT-LEFT EQUIVALENCE 155}

and

$H^{-1}:\left(\mathbb{R}_{y}^{p} \times \mathbb{R}_{y^{\prime}}^{q} \times \mathbb{R}_{\lambda}^{p} \times \mathbb{R}_{\lambda^{\prime}}^{q},(0,0,0,0)\right) \rightarrow\left(\mathbb{R}_{y}^{p} \times \mathbb{R}_{y^{\prime}}^{q} \times \mathbb{R}_{\lambda}^{p} \times \mathbb{R}_{\lambda^{\prime}}^{q},(0,0,0,0)\right)$

such that the following diagram commutes:

$$
\begin{array}{ccc}
\left(\mathbb{R}^{n} \times \mathbb{R}_{\lambda}^{p} \times \mathbb{R}_{\lambda^{\prime}}^{q},(0,0,0)\right) & \stackrel{h^{-1}}{\longrightarrow} & \left(\mathbb{R}^{n} \times \mathbb{R}_{\lambda}^{p} \times \mathbb{R}_{\lambda^{\prime}}^{q},(0,0,0)\right) \\
\left(\mathbb{R}_{y}^{p} \times \mathbb{R}_{y^{\prime}}^{q} \times \mathbb{R}_{\lambda}^{p} \times \mathbb{R}_{\lambda^{\prime}}^{q},(0,0,0,0)\right) & \stackrel{H^{-1}}{\longrightarrow} & \left(\mathbb{R}_{y}^{p} \times \mathbb{R}_{y^{\prime}}^{q} \times \mathbb{R}_{\lambda}^{p} \times \mathbb{R}_{\lambda^{\prime}}^{q},(0,0,0,0)\right) \\
\left(\mathbb{R}_{\lambda}^{p} \times \mathbb{R}_{\lambda^{\prime}, \lambda^{\prime}}^{q} \downarrow\right. \\
(0,0)) & = & \left(\mathbb{R}_{\lambda}^{p} \times \mathbb{R}_{\lambda^{\prime}}^{q},(0,0)\right) .
\end{array}
$$

We consider the inverse map germ $H$ of $H^{-1}$ and

$$
\phi_{H}^{\prime}=\left(\phi_{H, 1}^{\prime}, \phi_{H, 2}^{\prime}\right):\left(\mathbb{R}_{\lambda}^{p} \times \mathbb{R}_{\lambda^{\prime}}^{q},(0,0)\right) \rightarrow\left(\mathbb{R}_{y}^{p} \times \mathbb{R}_{y^{\prime}}^{q},(0,0)\right)
$$

associated with $H$.

Let $\Theta_{i}\left(t ;\left(\mathbf{y}, \mathbf{y}^{\prime}\right)\right) \quad\left(\right.$ resp. $\Theta_{j}^{\prime}\left(t ;\left(\mathbf{y}, \mathbf{y}^{\prime}\right)\right)$ ) be the integral curve of $\eta_{i}$ (resp. $\left.\eta_{j}^{\prime}\right)$ starting from $\left(\mathbf{y}, \mathbf{y}^{\prime}\right)$ and of time $t$. Then we can get the image $\phi_{H}^{\prime}\left(\lambda, \lambda^{\prime}\right)$ of $\left(\lambda, \lambda^{\prime}\right)=\left(\lambda_{1}, \ldots, \lambda_{p}, \lambda_{1}^{\prime}, \ldots, \lambda_{q}^{\prime}\right)$ by $\phi_{H}^{\prime}$ as the unique solution of the integral equation

(5.7) $\Theta_{1}\left(\lambda_{1} ; \ldots ; \Theta_{p}\left(\lambda_{p} ; \Theta_{1}^{\prime}\left(\lambda_{1}^{\prime} ; \ldots ; \Theta_{q}^{\prime}\left(\lambda_{q}^{\prime} ; \phi_{H}^{\prime}\left(\lambda_{1}, \ldots, \lambda_{p}, \lambda_{1}^{\prime}, \ldots, \lambda_{q}^{\prime}\right)\right) \ldots\right)=0\right.\right.$

By differentiating (5.7) with respect to $\lambda_{i}$ and $\lambda_{j}^{\prime}$, we get

$$
\eta_{i}\left(\Theta _ { i + 1 } \left(\lambda_{i+1} ; \ldots ; \Theta_{p}\left(\lambda_{p} ; \Theta_{1}^{\prime}\left(\lambda_{1}^{\prime} ; \ldots ; \Theta_{q}^{\prime}\left(\lambda_{q}^{\prime} ; \phi_{H}^{\prime}\left(\lambda, \lambda^{\prime}\right) \ldots\right)\right.\right.\right.\right.
$$$$
+\left(d \Theta_{1}\right)_{\left(y, y^{\prime}\right)} \ldots\left(d \Theta_{1}^{\prime}\right)_{\left(y, y^{\prime}\right)} \ldots\left(d \Theta_{q}^{\prime}\right)_{\left(y, y^{\prime}\right)} \partial^{q} \phi_{H}^{\prime}\left(\lambda, \lambda^{\prime}\right) / \partial \lambda_{i}=\mathbf{0}
$$

for any $i(1 \leq i \leq p)$ and

$$
\begin{aligned}
& \eta_{j}^{\prime}\left(\Theta _ { j + 1 } ^ { \prime } \left(\lambda_{j+1}^{\prime} ; \ldots ; \Theta_{q}^{\prime}\left(\lambda_{q}^{\prime} ; \phi_{H}^{\prime}\left(\lambda, \lambda^{\prime}\right) \ldots\right)\right.\right. \\
& +\quad\left(d \Theta_{1}\right)_{\left(y, y^{\prime}\right) \ldots\left(d \Theta_{1}^{\prime}\right)_{\left(y, y^{\prime}\right)} \ldots\left(d \Theta_{q}^{\prime}\right)_{\left(y, y^{\prime}\right)} \partial \phi_{H}^{\prime}\left(\lambda, \lambda^{\prime}\right) / \partial \lambda_{j}^{\prime}=\mathbf{0}}
\end{aligned}
$$

for any $j(1 \leq j \leq q)$.

Taking values at $\left(\lambda, \lambda^{\prime}\right)=(0,0)$ in $(5.8)$ and $(5.9)$, we get

$$
\begin{aligned}
\frac{\partial \phi_{H}^{\prime}}{\partial \lambda_{i}}(0,0) & =-\eta_{i}(0,0,0,0) \\
& =\mathbf{e}_{i} \quad(\text { by }(5.3))
\end{aligned}
$$


for any $i(1 \leq i \leq p)$ and

$$
\begin{aligned}
\frac{\partial \phi_{H}^{\prime}}{\partial \lambda_{j}^{\prime}}(0,0) & =-\eta_{j}^{\prime}(0,0,0,0) \\
& =\mathbf{e}_{p+j} \quad(\text { by }(5.4))
\end{aligned}
$$

for any $j(1 \leq j \leq q)$. Thus, $\phi_{H}^{\prime}$ is $C^{\infty}$ diffeomorphic. Therefore, by lemma (1.4), $f=\left(f_{1}, f_{2}\right)$ and $g=\left(g_{1}, g_{2}\right)$ are right-left equivalent.

Furthermore, by (5.5) and (5.7) we see

$$
\phi_{H, 2}^{\prime}(\lambda, 0)=0
$$

and by (5.6) and (5.7) we see

$$
\phi_{H, 1}^{\prime}\left(0, \lambda^{\prime}\right)=0 \text {. }
$$

(5.10) and (5.11) shows that

$$
\phi_{H}^{\prime} \text { maps } \mathbb{R}_{\lambda}^{p} \times\{0\} \quad\left(\text { resp. }\{0\} \times \mathbb{R}_{\lambda^{\prime}}^{p}\right)
$$$$
\text { to } \mathbb{R}_{y}^{p} \times\{0\} \quad\left(\text { resp. }\{0\} \times \mathbb{R}_{y^{\prime}}^{q}\right) \text {. }
$$

Thus, by (5.12) we see that $f=\left(f_{1}, f_{2}\right)$ and $g=\left(g_{1}, g_{2}\right)$ are in fact equivalent over varieties.

$\square$

\section{Proof of Theorem (3.2)}

Let $\Psi=\left(\Psi_{1}, \Psi_{2}\right):\left(\mathbb{R}^{n} \times \mathbb{R}_{\lambda}^{p} \times \mathbb{R}_{\lambda^{\prime}}^{q},(0,0,0)\right) \rightarrow\left(\mathbb{R}_{y}^{p} \times \mathbb{R}_{y^{\prime}}^{q},(0,0)\right)$ be the $C^{\infty}$ deformation of $f$ having the following form:

$$
\left[\begin{array}{c}
\Psi_{1}\left(x, \lambda, \lambda^{\prime}\right) \\
\Psi_{2}\left(x, \lambda, \lambda^{\prime}\right)
\end{array}\right]=\left[\begin{array}{c}
f_{1}(x) \\
f_{2}(x)
\end{array}\right]-N(x)\left[\begin{array}{c}
\lambda \\
\lambda^{\prime}
\end{array}\right] .
$$

Since

$$
\frac{\partial \Psi}{\partial \lambda_{i}}=-\mathbf{n}_{i}(x)=-\mathbf{e}_{i} \text { and } \frac{\partial \Psi}{\partial \lambda_{j}^{\prime}}=-\mathbf{n}_{p+j}(x)
$$

for any $i(1 \leq i \leq p)$ and any $j(1 \leq j \leq q)$, by lemma (4.4) we can choose germs of $C^{\infty}$ vector fields

$$
\begin{aligned}
& \tilde{\xi}_{i}, \tilde{\xi}_{j}^{\prime} \in m_{x} \mathcal{E}_{x, \lambda, \lambda^{\prime}}^{n}, \quad \text { and } \\
& \tilde{\eta}_{i}, \tilde{\eta}_{j}^{\prime} \in \mathcal{E}_{y, y^{\prime}, \lambda, \lambda^{\prime}}^{p+q}
\end{aligned}
$$

such that

\section{A CONSTRUCTIVE METHOD TO GET RIGIIT-LEFT EQUIVALENCE 157}

$$
\begin{gathered}
-\frac{\partial \Psi}{\partial \lambda_{i}}=-\tilde{\eta}_{i} \circ\left(\Psi_{1}, \Psi_{2}, \pi_{\lambda}, \pi_{\lambda^{\prime}}\right), \\
-\frac{\partial \Psi}{\partial \lambda_{j}^{\prime}}=\tilde{\xi}_{j}^{\prime}(\Psi)-\tilde{\eta}_{j}^{\prime} \circ\left(\Psi_{1}, \Psi_{2}, \pi_{\lambda}, \pi_{\lambda^{\prime}}\right), \\
\tilde{\eta}_{i}\left(y, y^{\prime}, \lambda, \lambda^{\prime}\right)=\mathbf{e}_{i}, \\
\tilde{\eta}_{j}^{\prime}(0,0,0,0)=\frac{\partial \Psi}{\partial \lambda_{j}^{\prime}}(0,0,0)=\mathbf{e}_{p+j}, \\
\tilde{\eta}_{j}^{\prime}\left(y, y^{\prime}, \lambda, \lambda^{\prime}\right) \text { depends only on } y^{\prime}, \lambda^{\prime}
\end{gathered}
$$

for any $i(1 \leq i \leq p)$ and any $j(1 \leq j \leq q)$.

By (6.1) and (6.2), integrating germs of $C^{\infty}$ vector fields

$$
\begin{aligned}
& \tilde{\xi}_{1}+\partial / \partial \lambda_{1}, \ldots, \tilde{\xi}_{p}+\partial / \partial \lambda_{p}, \\
& \tilde{\xi}_{1}^{\prime}+\partial / \partial \lambda_{1}^{\prime}, \ldots, \tilde{\xi}_{q}^{\prime}+\partial / \partial \lambda_{q}^{\prime} \\
& \tilde{\eta}_{1}+\partial / \partial \lambda_{1}, \ldots, \tilde{\eta}_{p}+\partial / \partial \lambda_{p}
\end{aligned}
$$

and

$$
\tilde{\eta}_{1}^{\prime}+\partial / \partial \lambda_{1}^{\prime}, \ldots, \tilde{\eta}_{q}^{\prime}+\partial / \partial \lambda_{q}^{\prime}
$$

yields $C^{\infty}$ diffeomorphic map germs

$$
\tilde{h}^{-1}:\left(\mathbb{R}^{n} \times \mathbb{R}_{\lambda}^{p} \times \mathbb{R}_{\lambda^{\prime}}^{q},(0,0,0)\right) \rightarrow\left(\mathbb{R}^{n} \times \mathbb{R}_{\lambda}^{p} \times \mathbb{R}_{\lambda^{\prime}}^{q},(0,0,0)\right)
$$

and

$\widetilde{H}^{-1}:\left(\mathbb{R}_{y}^{p} \times \mathbb{R}_{y^{\prime}}^{q} \times \mathbb{R}_{\lambda}^{p} \times \mathbb{R}_{\lambda^{\prime}}^{q},(0,0,0,0)\right) \rightarrow\left(\mathbb{R}_{y}^{p} \times \mathbb{R}_{y^{\prime}}^{q} \times \mathbb{R}_{\lambda}^{p} \times \mathbb{R}_{\lambda^{\prime}}^{q},(0,0,0,0)\right)$ such that the following diagram commutes:

$$
\begin{gathered}
\left(\mathbb{R}^{n} \times \mathbb{R}_{\lambda}^{p} \times \mathbb{R}_{\lambda^{\prime}}^{q},(0,0,0)\right) \\
\left(\Phi, \pi_{\lambda, \lambda^{\prime}}\right) \downarrow
\end{gathered} \stackrel{\tilde{h}^{-1}}{\longrightarrow} \quad\left(\mathbb{R}^{n} \times \mathbb{R}_{\lambda}^{p} \times \mathbb{R}_{\lambda^{\prime}}^{q},(0,0,0)\right)
$$

$\left(\mathbb{R}_{y}^{p} \times \mathbb{R}_{y^{\prime}}^{q} \times \mathbb{R}_{\lambda}^{p} \times \mathbb{R}_{\lambda^{\prime}}^{q},(0,0,0,0)\right) \stackrel{\widetilde{H}^{-1}}{\longrightarrow}\left(\mathbb{R}_{y}^{p} \times \mathbb{R}_{y^{\prime}}^{q} \times \mathbb{R}_{\lambda}^{p} \times \mathbb{R}_{\lambda^{\prime}}^{q},(0,0,0,0)\right)$

$$
\left(\mathbb{R}_{\lambda}^{p} \times \mathbb{R}_{\lambda^{\prime}}^{\pi_{\lambda, \lambda^{\prime}} \downarrow}(0,0)\right) \quad=\quad\left(\mathbb{R}_{\lambda}^{p} \times \mathbb{R}_{\lambda^{\prime}}^{\pi_{\lambda, \lambda^{\prime}}} \downarrow(0,0)\right) .
$$

We consider the inverse map germ $\widetilde{H}$ of $\widetilde{H}^{-1}$ and

$$
\phi_{\widetilde{H}}^{\prime}=\left(\phi_{\widetilde{H}, 1}^{\prime}, \phi_{\widetilde{H}, 2}^{\prime}\right):\left(\mathbb{R}_{\lambda}^{p} \times \mathbb{R}_{\lambda^{\prime}}^{q},(0,0)\right) \rightarrow\left(\mathbb{R}_{y}^{p} \times \mathbb{R}_{y^{\prime}}^{q},(0,0)\right)
$$


associated with $\widetilde{H}$.

Let $\tilde{\Theta}_{i}\left(t ;\left(\mathbf{y}, \mathbf{y}^{\prime}\right)\right) \quad\left(\right.$ resp. $\tilde{\Theta}_{j}^{\prime}\left(t ;\left(\mathbf{y}, \mathbf{y}^{\prime}\right)\right)$ be the integral curve of $\tilde{\eta}_{i}$ (resp. $\left.\tilde{\eta}_{j}^{\prime}\right)$ starting from $\left(\mathbf{y}, \mathbf{y}^{\prime}\right)$ and of time $t$. Then we can get the image $\phi_{\widetilde{H}}^{\prime}\left(\lambda, \lambda^{\prime}\right)$ of $\left(\lambda, \lambda^{\prime}\right)=\left(\lambda_{1}, \ldots, \lambda_{p}, \lambda_{1}^{\prime}, \ldots, \lambda_{q}^{\prime}\right)$ by $\phi_{\widetilde{H}}^{\prime}$ as the unique solution of the integral equation

(6.7) $\widetilde{\Theta}_{1}\left(\lambda_{1} ; \ldots ; \widetilde{\Theta}_{p}\left(\lambda_{p} ; \widetilde{\Theta}_{1}^{\prime}\left(\lambda_{1}^{\prime} ; \ldots ; \widetilde{\Theta}_{q}^{\prime}\left(\lambda_{q}^{\prime} ; \phi_{\widetilde{H}}^{\prime}\left(\lambda_{1}, \ldots, \lambda_{p}, \lambda_{1}^{\prime}, \ldots, \lambda_{q}^{\prime}\right)\right) \ldots\right)=0\right.\right.$.

By differentiating (6.7) with respect to $\lambda_{i}$ and $\lambda_{j}^{\prime}$, we get

$$
\tilde{\eta}_{i}\left(\widetilde { \Theta } _ { i + 1 } \left(\lambda_{i+1} ; \ldots ; \tilde{\Theta}_{p}\left(\lambda_{p} ; \widetilde{\Theta}_{1}^{\prime}\left(\lambda_{1}^{\prime} ; \ldots ; \tilde{\Theta}_{q}^{\prime}\left(\lambda_{q}^{\prime} ; \phi_{\widetilde{H}}^{\prime}\left(\lambda, \lambda^{\prime}\right) \ldots\right)\right.\right.\right.\right.
$$$$
+\left(d \widetilde{\Theta}_{1}\right)_{\left(y, y^{\prime}\right)} \ldots\left(d \tilde{\Theta}_{1}^{\prime}\right)_{\left(y, y^{\prime}\right)} \ldots\left(d \widetilde{\Theta}_{q}^{\prime}\right)_{\left(y, y^{\prime}\right)} \partial \phi_{\widetilde{H}}^{\prime}\left(\lambda, \lambda^{\prime}\right) / \partial \lambda_{i}=0
$$

for any $i(1 \leq i \leq p)$ and

$$
\begin{aligned}
& \tilde{\eta}_{j}^{\prime}\left(\widetilde { \Theta } _ { j + 1 } ^ { \prime } \left(\lambda_{j+1}^{\prime} ; \ldots ; \tilde{\Theta}_{q}^{\prime}\left(\lambda_{q}^{\prime} ; \phi_{\widetilde{H}}^{\prime}\left(\lambda, \lambda^{\prime}\right) \ldots\right)\right.\right. \\
& +\left(d \widetilde{\Theta}_{1}\right)_{\left(y, y^{\prime}\right)} \ldots\left(d \tilde{\Theta}_{1}^{\prime}\right)_{\left(y, y^{\prime}\right)} \ldots\left(d \tilde{\Theta}_{q}^{\prime}\right)_{\left(y, y^{\prime}\right)} \partial \phi_{\widetilde{H}}^{\prime}\left(\lambda, \lambda^{\prime}\right) / \partial \lambda_{j}^{\prime}=0
\end{aligned}
$$

for any $j(1 \leq j \leq q)$.

Taking values at $\left(\lambda, \lambda^{\prime}\right)=(0,0)$ in $(6.8)$ and $(6.9)$, we get

$$
\begin{aligned}
\frac{\partial \phi_{\tilde{H}}^{\prime}}{\partial \lambda_{i}}(0,0) & =-\eta_{i}(0,0,0,0) \\
& =\mathbf{e}_{i} \quad(\text { by }(6.3))
\end{aligned}
$$

for any $i(1 \leq i \leq p)$ and

$$
\begin{aligned}
\frac{\partial \phi_{\tilde{H}}^{\prime}}{\partial \lambda_{j}^{\prime}}(0,0) & =-\eta_{j}^{\prime}(0,0,0,0) \\
& =\mathbf{e}_{p+j} \quad(\text { by }(6.4))
\end{aligned}
$$

for any $j(1 \leq j \leq q)$. Thus, $\phi_{\widetilde{H}}^{\prime}$ is $C^{\infty}$ diffeomorphic. Therefore, by lemma (1.4), $f=\left(f_{1}, f_{2}\right)$ and $g=\left(g_{1}, g_{2}\right)$ are right-left equivalent.

Furthermore, by (6.5) and (6.7) we see

$$
\phi_{\widetilde{H}, 2}^{\prime}\left(\lambda, \lambda^{\prime}\right) \text { depends only on } \lambda^{\prime},
$$

and by (6.3), (6.5) and (6.7) we see

$$
\phi_{\widetilde{H}, 1}^{\prime}\left(\lambda, \lambda^{\prime}\right)=\lambda+\phi_{\widetilde{H}, 1}^{\prime}\left(0, \lambda^{\prime}\right) .
$$

(6.10) and (6.11) shows that $f=\left(f_{1}, f_{2}\right)$ and $g=\left(g_{1}, g_{2}\right)$ are in fact $\mathcal{R}^{+}$. equivalent.

\section{References}

[AGV ] V.I.Arnol'd, S.M.Gusein-Zade, A.N.Varchenko, "Singularities of Differentiable Maps I," Birkhauser, 1985

[D1 ] J.P. Dufour, Sur la stabilité de diagrammes d'applications différentiables, Ann. Sc. Ec. Norm. Sup. 10 (1977), 153-174.

[D2 ] Families de courbes planes différentiables, Topology 22 (1983), $449-474$.

[dP ] A. A. du Plessis, On the determinacy of smooth map-germs, Inventiones math. 58 (1980), 107-160.

[Ga ] T. Gaffney, A note on the order of determination of a finitely determined germ, Inventiones math. $\mathbf{5 2}$ (1979), 127-130.

[HIIY ] A. Hayakawa, G. Ishikawa, S. Izumiya, K. Yamaguchi, Classification of integral diagrams of Whitney type and first order differential equations, preprint.

[Mg ] B. Malgrange "Ideals of Differentiable Functions," Oxford Univ. Press, 1966.

[Mr ] J. Martinet, Deploiements versels des applications differentiables et classification des applications stables, in "Singularites d'Applications Differentiables, Plans-Sur-Bex," Springer Lecture Notes in Mathematics 535, 1976, pp. 1-44

[M1 ] J. Mather, Stability of $C^{\infty}$ mappings, III, Finitely determined map germs, Publ. Math. I.H.E.S. 35 (1969), 127-156.

[M2 ] Stability of $C^{\infty}$ mappings, $I V$, Classification of stable germs by $\mathbb{R}$ algebras, Publ. Math. I.H.E.S. 37 (1970), 223-248.

[Na1 ] I. Nakai, On diagrams of smooth maps, RIMSkokyuroku 725 (1990), 117-124. 
[Na2 ] - Notes on versal deformation of first order PDE and web structure, preprint.

[N ] T. Nishimura, Isomorphism of smooth map germs with isomorphic local algembras, preprint.

[W ] C. T. C. Wall, Finite determinacy of smooth map-germs, Bull. London Math. Soc. 13 (1981), 481-539.

Departamento de Matemática

Faculty of Education

Yokohoma National University

Yokohama 240, JAPAN 